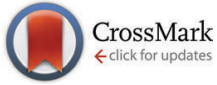

Cite this: Phys. Chem. Chem. Phys., 2015, 17, 5314

Received 5th November 2014, Accepted 9th January 2015

DOI: 10.1039/c4cp05116e

www.rsc.org/pccp

\section{Calculating nuclear magnetic resonance shieldings using systematic molecular fragmentation by annihilation $\dagger$}

\author{
David M. Reid and Michael A. Collins \\ SMFA was used to calculate NMR shieldings in a test set of 15 molecules. Level 4 fragments were found \\ to yield satisfactory results when hydrogen bonding was included in the calculations. The utility of \\ additional long range corrections was also investigated. It was found that with hydrogen bonding already \\ included, ab initio long range corrections were not necessary. Instead, inclusion of the McConnell \\ correction for fragments was found to be sufficient. With these parameters the algorithm produces \\ MADs of $0.046,0.26,0.24$ and 1.04 ppm for hydrogens, carbons, nitrogens and oxygens respectively.
}

\section{Introduction}

It is difficult to overemphasise the importance of NMR in chemistry. Since its development it has become a standard technique for determining or confirming the structure of novel molecules, and has also seen wide usage in the study of dynamic properties. For many systems of interest, however, their size precludes the accurate determination of structural parameters, with peak broadening and overlap leading to ambiguities in data interpretation.

For this reason, theoretical methods are often useful in resolving these ambiguities, though these suffer from similar drawbacks as the system size increases. $\operatorname{CCSD}(\mathrm{T})$ is widely considered the gold standard of theoretical calculations for NMR Shieldings, ${ }^{1}$ though its $\mathrm{N}^{7}$ scaling in computational cost with system size limits its use to the study of molecules with no more than 4 or 5 heavy atoms. The $\mathrm{N}^{3}$ scaling of many DFT functionals solves this problem, though at a considerable cost in accuracy, and while there exist functionals that have been parameterised specifically for $\mathrm{NMR}^{2,3}$ the utility of these functionals for assignment in novel compounds is questionable. ${ }^{4}$

Amongst the most promising approaches for reducing the cost of theoretical calculations are linear scaling methods, in particular fragmentation. These are well suited for NMR calculations as NMR is a local property and such algorithms preserve the local environment in which nuclei reside. A variety of such methods have already been applied to the calculation of NMR

Research School of Chemistry, The Australian National University, ACT, Australia. E-mail:dreid@rsc.anu.edu.au,collins@rsc.anu.edu.au

$\dagger$ Electronic supplementary information (ESI) available: Coordinates and illustrations for the 15 molecules on which calculations were performed in this work. Additional data on the CPU times for the full molecule and SMFA Level 4 calculations is also included. See DOI: $10.1039 / \mathrm{c} 4 \mathrm{cp} 05116 \mathrm{e}$ shieldings, including the Isodesmic ${ }^{5}$ and the Combined Fragmentation Methods (CFM), ${ }^{6}$ a density matrix based algorithm referred to as GIAO-HF, ${ }^{7-11}$ Automatic Fragment Quantum Mechanics/Molecular Mechanics (AF-QM/MM), ${ }^{12-15}$ Adjustable Density Matrix Assembler (ADMA) ${ }^{16-18}$ and the Fragment Molecular Orbital (FMO) method. ${ }^{19,20}$

Of the energy-based fragmentation schemes, systematic molecular fragmentation by annihilation (SMFA) provides an accurate description of molecular energies in terms of the energies of relatively small molecular fragments. Small fragment size is important if high levels of ab initio theory, such as $\operatorname{CCSD}(\mathrm{T})$, are to be employed to calculate chemical shieldings in large molecules. The aim of this paper is to establish the accuracy of SMFA for calculations of chemical shieldings. SMFA is systematic in the sense that energies and other properties (including chemical shieldings) can be evaluated in a hierarchy of "Levels" which correspond to increasing fragment sizes. Hence, the convergence of the accuracy of SMFA chemical shieldings can be evaluated as a function of "Level", or fragment size.

In addition, some of the aforementioned fragmentation algorithms incorporate a long range $a b$ initio non-bonded correction to improve the accuracy of the reported shielding. The feasibility of replacing all or part of this additional $a b$ initio calculation with a perturbative long range correction is investigated.

The paper is set out as follows. Section 2 presents a brief description of the SMFA method as applied to chemical shieldings, and details including a description of the set of molecules employed to test the accuracy of these calculations. Section 3 presents the results, which indicate the accuracy and reliability of these calculations. The paper finishes with a brief discussion. 


\section{Method}

\subsection{Fragmentation}

The chemical shielding tensor of a nucleus can be calculated as:

$$
\sigma_{\alpha \beta}^{n}=\frac{\partial^{2} E}{\partial \mu_{\alpha}^{n} \partial B_{\beta}}
$$

where $\sigma_{\alpha \beta}^{n}$ is a component of the shielding tensor $\overline{\bar{\sigma}}$ for atom $n, E$ is the energy of the molecule, $\mu_{\alpha}^{n}$ is the component of magnetic moment of nucleus $n$ in the $\alpha$ direction, and $B_{\beta}$ is the component of the applied magnetic field in the $\beta$ direction.

The chemical shielding can then be obtained by taking the isotropic component of this tensor:

$$
\sigma^{n}=\frac{1}{3} \sum_{\alpha} \sigma_{\alpha \alpha}^{n}
$$

In this work, the molecular energy, and subsequently the chemical shielding, are evaluated using the systematic molecular fragmentation by annihilation (SMFA) approximation. As this approximation has been described in detail elsewhere [see ref. 21 and 22 and references therein], only a brief outline will be given here.

2.1.1 Bonded interactions. The approach begins by considering a molecule as a set of functional groups connected by single bonds. The algorithm allows the user to include hydrogen bonds as single bonds, or not. The molecule is then decomposed into fragments by removing functional groups in an automated sequence of steps that preserves the bonding environment of each group to some extent. When groups are eliminated in the fragmentation procedure, the remaining groups have unsatisfied valency. The normal valency of each atom is restored by appending hydrogen atoms along the original bond direction, as previously described. ${ }^{23}$ The method has a systematic set of "Levels" which determine the proximity of eliminated groups, so that with increasing Level, a more extensive bonding environment is retained. At Level 1, the interaction of each group with its $\alpha$ substituents is included in the fragments. At Level 2, all $\beta$ substituent interactions are included, and so on.

In this scheme, any general molecule can be decomposed as:

$$
M \rightarrow \sum_{i=1}^{N_{\text {frag }}} c_{i} F_{i}
$$

where $F_{i}$ are overlapping fragments, while $c_{i}$ are the integer fragment coefficients. The energy of the molecule is then given by:

$$
E_{\mathrm{b}}=\sum_{i=1}^{N_{\text {frag }}} c_{i} E\left(F_{i}\right)
$$

where the energy has been denoted $E_{\mathrm{b}}$ to indicate that only nearby through bond interactions have been included. The composition of the fragments in eqn (3) are determined by the bonding between groups. If hydrogen bonds are included as single bonds, these fragments may contain groups connected by hydrogen bonds.

2.1.2 Non-bonded interactions. A better description of the molecular energy can be obtained if non-bonded interactions are included:

$$
E=E_{\mathrm{b}}+E_{\mathrm{nb}}
$$

The SMFA algorithm treats non-bonded interactions as follows: first the molecule is fragmented at two given Levels, denoted LX and LY. Each LX fragment is then checked against each LY fragment to determine if their constituent groups have appeared together at the original Level of fragmentation. To avoid double counting, interactions that have already been accounted for in eqn (4) are screened out or modified, as described previously. ${ }^{23}$ With this modification, the non-bonded energy is written as:

$$
E_{\mathrm{nb}}=\frac{1}{2} \sum_{i=1}^{N_{\text {frag }}} \sum_{j=1}^{N_{\text {frag }}} c_{i} c_{j}\left(E\left(F_{i} F_{j}\right)-E\left(F_{i}\right)-E\left(F_{j}\right)\right)
$$

where the indices $i$ and $j$ run over all LX and LY fragments respectively.

The allowed interaction pairs are divided into two categories: short and long range. Interactions that are deemed short range are treated $a b$ initio, while those that are considered long range are treated perturbatively. The cutoff between long and short is controlled by a distance based parameter, $d_{\text {tol }}$. For each LX-LY pair the minimum ratio of the atom-atom distance to van der Waals radii $\left(r_{\mathrm{vdw}}\right)$, denoted $d_{\mathrm{vdW}}$, is determined as:

$$
d_{\mathrm{vdW}}=\min \left(\frac{\left\|\vec{r}_{m}-\vec{r}_{n}\right\|}{r_{\mathrm{vdW}_{m}}+r_{\mathrm{vdW}_{n}}}\right)
$$

where the indices $m$ and $n$ run over all atoms in the LX and LY fragments respectively. If this is less than the cutoff $d_{\text {tol }}$, then the interaction is considered short range. Determination of an optimum value for $d_{\text {tol }}$ is one of the goals of this work.

Another important parameter is the size of the interacted fragments. Larger fragments should give more accurate interactions, though at a significantly increased cost. In this work, setting both LX and LY to Level 1 was found to be the best compromise between accuracy and computational cost.

The interaction of pairs of fragments that are separated by more than $d_{\text {tol }}$ also make a contribution to the total molecular energy, and hence a contribution to the chemical shielding tensor, via eqn (1). In applications of SMFA to the evaluation of molecular energies, these long range fragment-fragment interactions are evaluated using perturbation theory. ${ }^{24}$ Here the contributions of these long range interactions to the chemical shielding tensors of the fragment nuclei can be directly evaluated using perturbation theory. These perturbative contributions are commonly denoted as "McConnell corrections". 25

Each fragment, $F_{j}$, has a magnetic susceptibility tensor, $\overline{\bar{\chi}}_{j}$. An external magnetic field induces a magnetic field proportional to $\overline{\bar{\chi}}_{j}$, which in turn produces a correction to the chemical shielding tensor in atom $n$ in fragment $i$. If fragments $j$ and $i$ are well separated, the McConnell correction $\left(\delta \overline{\bar{\sigma}}^{n}\right)$ is:

$$
\delta \overline{\bar{\sigma}}^{n}=\frac{\overline{\bar{\chi}}_{j}}{r_{n j}{ }^{3}}-\frac{\overline{\bar{\chi}}_{j} \cdot \vec{r}_{n j} \cdot \vec{r}_{n j}^{T}}{r_{n j}{ }^{5}}
$$

where $\vec{r}_{n j}$ is the vector connecting atom $n$ (in fragment $i$ ) with fragment $j$. There is some arbitrariness in the choice of origin for fragment $j$. For this work we have proceeded as recommended in 
ref. 25 and chosen the centre of nuclear charge of fragment $j$ to be the origin for the purposes of this equation.

Adding the McConnell correction to the terms already described gives a total equation for the shielding of nucleus $n$ :

$$
\begin{aligned}
\sigma^{n}= & \sum_{i=1}^{N_{\text {frag }}} c_{i} \sigma^{n}\left(F_{i}\right) \\
& +\sum_{i=1}^{N_{\text {frag }}} \sum_{j=1 ; d_{\mathrm{vdW}}<d_{\mathrm{tol}}}^{N_{\text {frag }}} c_{i} c_{j}\left(\sigma^{n}\left(F_{i} F_{j}\right)-\sigma^{n}\left(F_{i}\right)\right) \\
& +\sum_{i=1}^{N_{\text {frag }}} \sum_{j=1 ; d_{\mathrm{vdW}}>d_{\mathrm{tol}}}^{N_{\text {frag }}} \frac{1}{3} \operatorname{tr}\left(\frac{\overline{\bar{\chi}}_{j}}{r_{n j}{ }^{3}}-\frac{\overline{\bar{\chi}}_{j} \cdot \vec{r}_{n j} \cdot \vec{r}_{n j}^{T}}{r_{n j}{ }^{5}}\right)
\end{aligned}
$$

where nucleus $n$ is in fragment $i$ but not fragment $j$, and $t r$ denotes the trace of the tensor.

2.1.3 Embedded charges. It has been demonstrated for a number of fragmentation methods ${ }^{20,26-29}$ that the $a b$ initio energies in eqn (4) and (6) provide more accurate estimates of the total molecular energy when evaluated in the presence of embedded charges that represent the charge distribution in the remainder of the molecule. We have verified that similarly significant improvement in the accuracy of calculated chemical shielding tensors is obtained by using such embedded charges. The embedded charges used in this work have been evaluated using the method of earlier work ${ }^{30}$ with some modifications. The electron density of fragments for Level $=0$ (rather than higher values of Level, as in earlier work) was evaluated and represented by distributed multipoles on each atom, using Stone's method. ${ }^{31}$ Charges on capping hydrogen atoms were assigned to the heavy atoms to which they are bonded. Multipoles up to second order were employed. The efficacy of using natural population analysis charges ${ }^{32,33}$ was also explored, but found to produce less accurate shieldings.

\subsection{Molecules chosen}

Calculations were performed on a collection of 15 moderate sized organic molecules with a variety of structures. The test set includes $\alpha$-helix and $\beta$-sheet like peptides as well as lipids, phospholipids, and sugars. These molecules contain between 47 and 110 atoms, with an average of 79 atoms per molecule. Amongst these there are 616 hydrogens, 368 carbons, 57 nitrogens and 131 oxygens. Four of these molecules contain formal charges: two molecules are zwitterions with two charged sites each $(+1$ and -1$)$, one is a zwitterion with four charged sites $(+1,-1,+1,-1)$ and one molecule is a dication with two charged sites $(+1,+1)$.

Coordinates for all molecules can be found in the ESI. $\dagger$ The coordinates of 12 of these molecules were obtained from the Cambridge Structural Database, ${ }^{34}$ and have not been modified. The coordinates of three molecules (denoted GelA, GelB and glycine in the $\mathrm{ESI} \dagger$ ) were obtained from ref. 35 as structures optimised at the HF/6-31G(d) level of theory. Pictorial representations of the structures of all 15 molecules are also contained in the ESI. $\dagger$ To assess the error associated with the fragmentation approximation, the shieldings calculated at each
Level of fragmentation were compared with those calculated for the full molecule at the same level of theory. Mean absolute deviations from the full molecule shieldings are evaluated over all nuclei of a given type across the 15 molecules in the test set.

\subsection{Computational details}

All calculations were performed using the Gaussian09 program package. $^{36}$ GIAO was used to ensure gauge invariance. Background multipoles were calculated up to second order using GDMA2. ${ }^{31}$ Calculations for all systems were performed $a b$ initio and in vacuo at $\mathrm{HF}$ with the $6-31++\mathrm{G}(\mathrm{d}, \mathrm{p})$ basis set.

It should be noted that this level of theory and basis set is not recommended for practical calculations. The accurate reproduction of experimental results would likely require calculations of at least MP2 quality with a quadruple zeta basis set. $^{1,4,37}$ However, the full molecule calculations required to gauge the accuracy of SMFA are not feasible at such a high level of $a b$ initio theory. Nonetheless, it is useful to investigate the accuracy of SMFA chemical shieldings for different basis sets and levels of theory.

To this end, additional calculations were performed at B3LYP/6-31++G(d,p), MP2/6-31G, HF/6-31G and HF/6-311++G(d,p) on 3 molecules from the test set: BAVCAC, ZEVHIR and GelB. These molecules were chosen as they are broadly representative of the molecules in this test set, while still being small enough for calculations to be feasible at the more expensive model chemistries. All shieldings are reported in parts per million (ppm).

\section{Results}

It should be noted that the accuracy required for reported shieldings depends upon the type of nuclei under consideration. While hydrogen shieldings typically appear within a range of approximately $10 \mathrm{ppm}$, carbon shieldings can differ by up to 200 ppm, and heavier nuclei, such as nitrogen and oxygen, can vary on even larger scales. Consequently, an accuracy of 0.1 ppm for hydrogens and $1.0 \mathrm{ppm}$ for carbons and nitrogens is considered satisfactory for the purpose of this study, as this represents $1 \%$, or less, of the total shielding scale.

\subsection{Interaction radii cutoff}

The impact of including through space interactions at varying cutoff radii is illustrated graphically in Fig. 1. Results are only displayed for hydrogens since the heavier nuclei exhibit similar trends. It is immediately evident from Fig. 1 that the inclusion of through space interactions only reduces the mean deviations if hydrogen bonding was not included in the original fragment calculations. This suggests that the improved errors at higher values of $d_{\text {tol }}$ in Fig. 1a are dominated by an improved description of $\sigma$ for atoms that are involved in hydrogen bonding. Inspection of shieldings for individual nuclei confirms that for hydrogens, nitrogens and oxygens, all significant improvements in the predicted shielding occurred for atoms involved in hydrogen bonding. For carbons this was not the case, and 

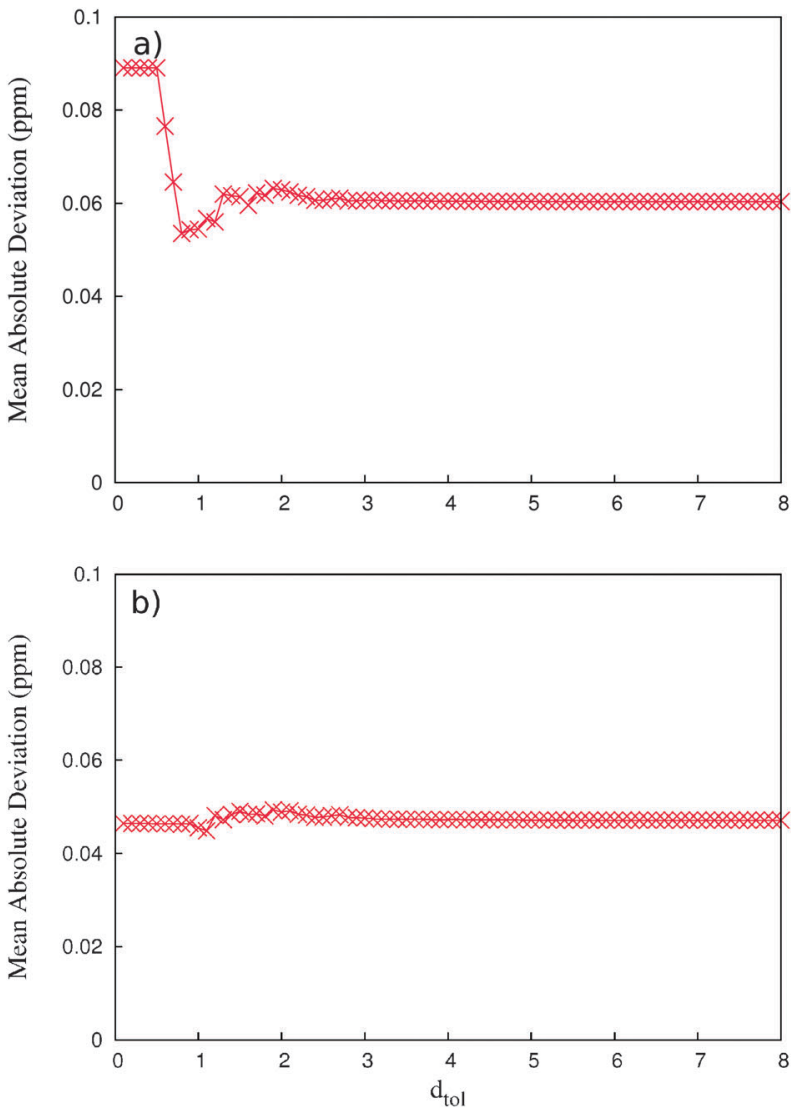

Fig. 1 Mean absolute deviations from full molecule calculations as a function of $d_{\text {tol }}$ for hydrogens at Level 4 . (a) Without and (b) with hydrogen bonding.

there was no clear pattern to the improvement of errors upon inclusion of hydrogen bonding.

Furthermore, atoms involved in hydrogen bonding display lower errors when hydrogen bonding is included in the fragment calculations than when it is treated through the non-bonded correction. This is to be expected since treating hydrogen bonds in a similar fashion to single bonds ensures that some fragments in eqn (3) contain hydrogen bonds with alpha, beta, gamma, etc. substituent groups, providing a more extensive description of the chemical environment of these bonds than would be the case if such interactions were included via eqn (6).

Overall, this suggests that the inclusion of background charges and the McConnell correction is adequate for all nonbonded interactions except hydrogen bonds. Furthermore, treating hydrogen bonding at the original Level of fragmentation appears to be more effective, with mean absolute deviations (MADs) lower at $d_{\text {tol }}=0$ in Fig. $1 \mathrm{~b}$ than at any value of $d_{\text {tol }}$ in Fig. 1a. It should be noted, however, that this can be partially ascribed to the larger average fragment size when hydrogen bonding is included. Based on this, we conclude that the best practice is to perform calculations without L1-L1 ab initio interactions (that is, $d_{\mathrm{tol}}=0$ ), and with hydrogen bonding treated in the base fragmentation calculation. Setting $d_{\mathrm{tol}}=0$ (the recommended value) means that all non-bonded contributions in eqn (9) are evaluated using the McConnell correction.
Table 1 Mean absolute deviations of the shielding constants from full molecule values are shown for hydrogen atoms, with and without the McConnell correction. Results are for $d_{\text {tol }}=0.0$ and hydrogen bonding included

\begin{tabular}{llllll}
\hline Level & 2 & 3 & 4 & 5 & 6 \\
\hline McConnell & 0.177 & 0.079 & 0.046 & 0.033 & 0.021 \\
No McConnell & 0.192 & 0.096 & 0.064 & 0.049 & 0.032
\end{tabular}

The utility of the McConnell correction is illustrated in Table 1, from which it can be seen that its inclusion reduces MADs for hydrogens by approximately $0.015 \mathrm{ppm}$ at all levels of fragmentation except Level 6 . The magnitude of the correction is similar for heavier atoms, though owing to the larger shielding scales for these nuclei, such a result is less significant. As the calculations necessary for performing the McConnell correction are considerably cheaper than those associated with the first term in eqn (9), it is worth including in all calculations for which hydrogen shieldings are of interest.

\subsection{Convergence with Level of fragmentation}

The convergence of shieldings with respect to Level of fragmentation is displayed for hydrogens, carbons, nitrogens and oxygens in Fig. 2. For all types of nuclei, the MADs converge approximately exponentially. However, this is not the case for
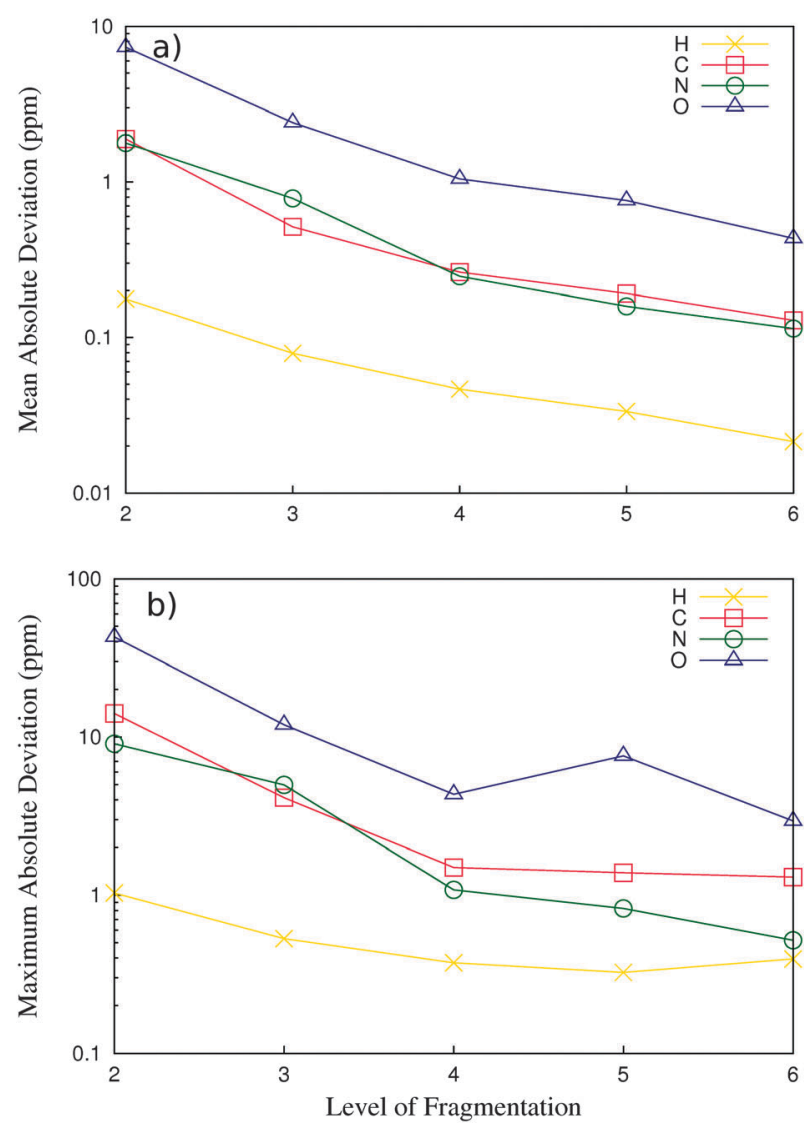

Fig. 2 (a) Mean absolute and (b) maximum absolute deviations from full molecule calculations. $d_{\text {tol }}=0.0$ and hydrogen bonding is included in calculations. 

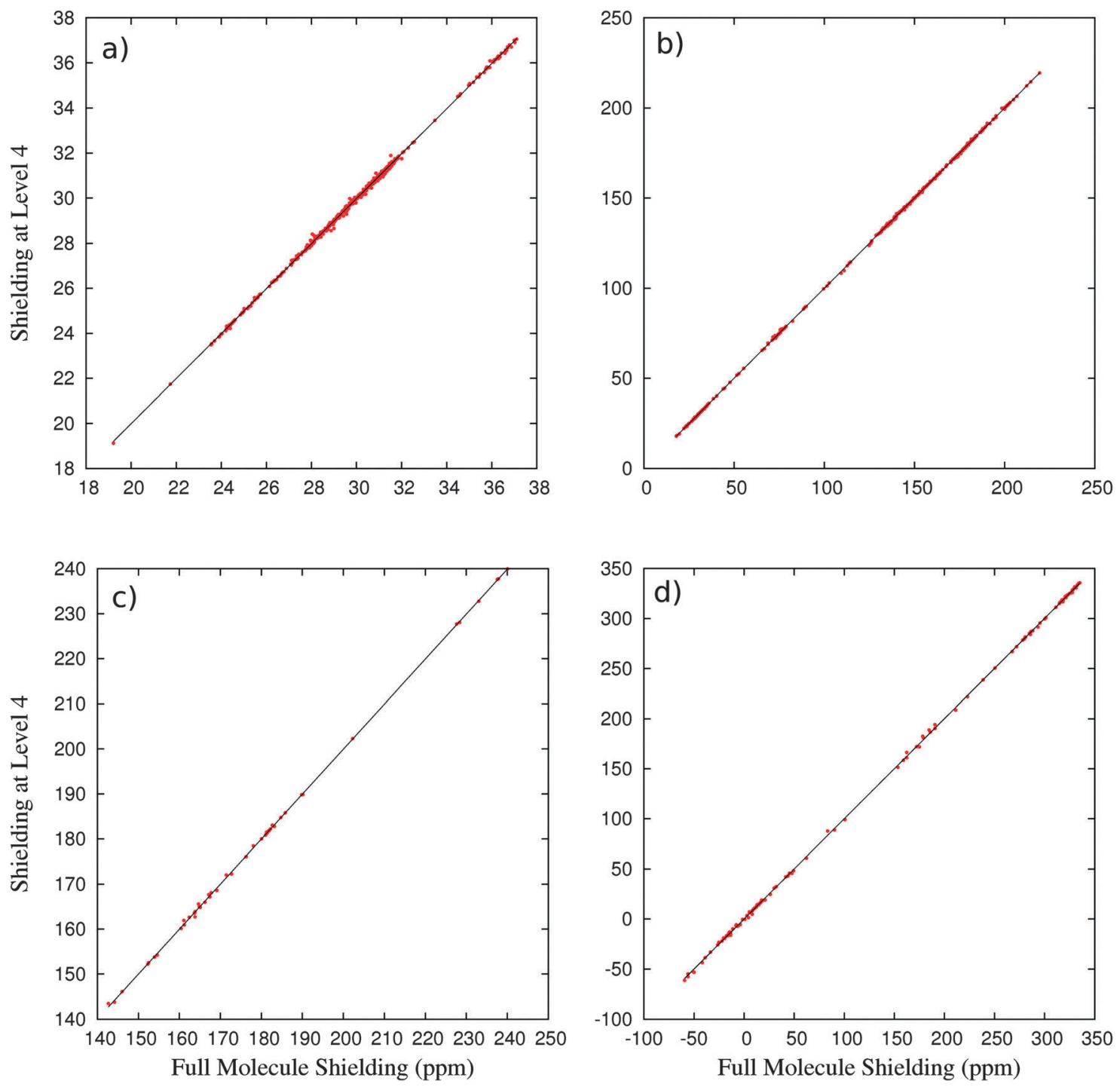

Fig. 3 Shieldings calculated at Level 4 with $d_{\mathrm{tol}}=0$ and hydrogen bonding included, are plotted against full molecule shieldings for all (a) hydrogens, (b) carbons, (c) nitrogens, and (d) oxygens in the test set of 15 molecules.

the maximum deviations. This demonstrates that convergence towards the full molecule results does not occur at the same rate, and is not necessarily monotonic, for all atoms.

A consequence of this is that a small difference between shieldings for a specific nucleus at two given Levels of fragmentation suggests, but does not guarantee, that either Level is a good approximation to the full molecule result. This further suggests that extrapolation of $\sigma$ with respect to Level of fragmentation will not guarantee reliable approximations to the full molecule shieldings.

Regardless, MADs for all types of nuclei are within the desired Level of accuracy by the time Level 4 fragmentation is reached, suggesting that this is a reliable Level for practical calculations.

It should also be noted that, from an assignment perspective, the ordering of nuclei with respect to each other is more important than the mean error. The extent to which this will be correctly reproduced can be assessed from the scatter plots, presented in Fig. 3. Inspecting these scatter plots it is evident that the most satisfactory results, in terms of relative ordering, are for carbons, for which the results form a near perfect straight line. Results are slightly less satisfactory for nitrogens and oxygens, though the correct order appears to be preserved. The only nuclei for which it is clear that misassignments may be made at this Level of fragmentation is hydrogens, for which the ordering is incorrect in a small number of cases.

\subsection{Level of theory and basis set dependence}

MADs for hydrogen shielding constants are displayed for the various level of theory and basis set combinations in Table 2 . The shielding constants for other nuclei exhibit similar trends and are hence not shown. The first three entries in Table 2 demonstrate that the accuracy of SMFA is largely uninfluenced by changes in the basis set. Likewise, the accuracy of SMFA at MP2 differs negligibly from that at HF. For B3LYP, the MAD is more than double the corresponding value at $\mathrm{HF}$, suggesting that, for this small sample set, SMFA is less accurate for DFT calculations of NMR shieldings. However, the MAD is below 
Table 2 Mean absolute deviations of the shielding constants from full molecule values are shown for the hydrogen atoms in BAVCAC, ZEVHIR and GelB, using various model chemistries

\begin{tabular}{ll}
\hline Model chemistry & Hydrogen MAD \\
\hline HF/6-31G & 0.042 \\
HF/6-31++G(d,p) & 0.038 \\
HF/6-311++G(d,p) & 0.036 \\
B3LYP/6-31++G(d,p) & 0.098 \\
MP2/6-31G & 0.043
\end{tabular}

$0.1 \mathrm{ppm}$, so that SMFA still represents a reasonable approximation for the shieldings calculated with B3LYP.

\subsection{Computational cost}

The CPU times for the full molecule and SMFA Level 4 calculations at $\mathrm{HF} / 6-31++\mathrm{G}(\mathrm{d}, \mathrm{p})$ are displayed in Table S16 of the ESI $\dagger$ for the 15 molecules in the test set. The corresponding results for SMFA Levels 3 and 4, at MP2/6-31G, are presented in Table 3 for BAVCAC, ZEVHIR and GelB. Table S16 (ESI $\dagger$ ) shows that, for a number of molecules, the $\mathrm{HF} / 6-31++\mathrm{G}(\mathrm{d}, \mathrm{p})$ Level 4 calculation proved more computationally expensive than the full molecule calculation. The large variation observed for SMFA CPU times in Table S16 (ESI $\dagger$ ) is due primarily to structural differences between the molecules in the test set, rather than to system size. In particular, the most expensive calculations are for molecules that produce the largest fragments. These are typically peptides with extensive hydrogen bonding, since amides are treated as a single group in SMFA and hydrogen bonding results in small ring structures. The large variation in CPU times can also be clearly seen in Table 3 for MP2/6-31G, where Level 4 calculations for ZEVHIR are more expensive than those for GelB by a factor of 50. This is despite GelB having 102 atoms while ZEVHIR has only 79 , and is due to the two molecules producing fragments with an average of 16.71 and 38.53 atoms respectively.

Nonetheless, the computational advantage of using fragmentation becomes more significant with larger systems or higher levels of theory. The latter point is again evident in Table 3, with even the most expensive SMFA calculation proving 2.5 times faster than its full molecule counterpart. One additional advantage of using SMFA is that an increase in system size will increase the number of calculations, but will not increase the size of the individual fragment calculations, thereby keeping the largest system calculations within the realm of feasibility. In addition, SMFA is readily amenable to parrallelization, since the calculations for each fragment are independent. As a consequence, each fragment calculation can be run on separate CPUs, and the effective wall time is simply

Table 3 The total CPU time (in hours) at MP2/6-31G is shown for BAVCAC, ZEVHIR and GelB, for the whole molecule calculation and SMFA Levels 3 and 4

\begin{tabular}{lccr}
\hline & \multicolumn{2}{l}{ CPU time } & \\
\cline { 2 - 4 } Molecule & Full molecule & Level 3 & Level 4 \\
\hline BAVCAC & 9.31 & 0.32 & 0.79 \\
ZEVHIR & 151.25 & 27.03 & 66.45 \\
GelB & 324.12 & 0.61 & 1.28
\end{tabular}

Table 4 The effective wall time (in hours) at MP2/6-31G is shown for BAVCAC, ZEVHIR and GelB, for the whole molecule calculation and SMFA Levels 3 and 4 . The maximum possible eight processors were used for each individual ab initio calculation. For SMFA calculations, the wall time is that for the largest fragment calculation

\begin{tabular}{llll}
\hline & \multicolumn{2}{l}{ Wall time } & \\
\cline { 2 - 4 } Molecule & Full molecule & Level 3 & Level 4 \\
\hline BAVCAC & 1.164 & 0.005 & 0.019 \\
ZEVHIR & 18.906 & 0.689 & 2.063 \\
GelB & 40.515 & 0.016 & 0.025
\end{tabular}

the CPU time of the largest fragment calculation. For the full molecule, the Gaussian09 calculation is only partly parallel, and limited to eight processors. The wall times for the full molecule and SMFA calculations are displayed in Table 4.

\section{Conclusions}

SMFA has been shown here to reproduce whole molecule values of NMR shielding constants for $\mathrm{H}, \mathrm{C}, \mathrm{N}$, and $\mathrm{O}$ atoms with MADs of 0.046, 0.26, 0.24 and $1.04 \mathrm{ppm}$, respectively, at Level 4 . This is comparable to previously reported $a b$ initio shielding constants obtained via fragmentation. ${ }^{6,12,13,16}$ Due mostly to the different ways that fragments are defined in different algorithms, SMFA generally produces the smallest fragments of all algorithms. The ability to produce comparable accuracy using smaller fragments is likely due to two factors; the inclusion of hydrogen bonding in the primary molecular fragmentation, and the use of the McConnell correction to describe all nonbonded effects. In addition to reducing the size of the fragments required for the determination of accurate shieldings, SMFA provides accurate calculations without $a b$ initio through space corrections. It should be noted, however, that the errors introduced by the fragmentation approximation are within acceptable limits for all algorithms.

Several obstacles must be overcome before the $a b$ initio calculation of chemical shieldings for large molecules in solution can accurately reproduce experimental results. As demonstrated herein and elsewhere, ${ }^{6-20}$ energy-based fragmentation reduces the size of the fragments/molecules for which calculations are necessary, without undue loss of accuracy. Recently, the use of locally dense basis sets has been shown to further reduce the computational task substantially, even for the relatively small molecular fragments produced by SMFA. ${ }^{38-40}$ Further computational savings will be necessary if the most reliable ab initio methods are to be employed. In addition, reproducing experimental shieldings would require corrections due to the solvent and counter-ions present in solution, conformational/torsional motion, and high frequency vibrations at equilibrium geometries, though some work has already been done in these areas. ${ }^{12-18}$

\section{References}

1 T. Kupka, M. Stachów, M. Nieradka, J. Kaminsky and T. Pluta, J. Chem. Theory Comput., 2010, 6, 1580-1589. 
2 K. W. Wiitala, T. R. Hoye and C. J. Cramer, J. Chem. Theory Comput., 2006, 2, 1085-1092.

3 T. W. Keal and D. J. Tozer, J. Chem. Phys., 2004, 121, 5654-5660.

4 A. M. Teale, O. B. Lutns, T. Helgaker, D. J. Tozer and J. Gauss, J. Chem. Phys., 2013, 138, 024111.

5 A. M. Lee and R. P. A. Bettens, J. Phys. Chem. A, 2007, 111, 5111-5115.

6 H.-J. Tan and R. P. A. Bettens, Phys. Chem. Chem. Phys., 2013, 15, 7541-7547.

7 C. Ochsenfeld, J. Kussman and F. Koziol, Angew. Chem., Int. Ed., 2004, 43, 4485-4489.

8 J. Kussman and C. Ochsenfeld, J. Chem. Phys., 2007, 127, 054103.

9 M. Beer, J. Kussmann and C. Ochsenfeld, J. Chem. Phys., 2011, 134, 074102.

10 D. Flaig, M. Beer and C. Ochsenfeld, J. Chem. Theory Comput., 2012, 8, 2260-2271.

11 M. Maurer and C. Ochsenfeld, J. Chem. Phys., 2013, 138, 174104.

12 X. He, B. Wang and K. M. Merz, Jr., J. Phys. Chem. B, 2009, 113, 10380-10388.

13 T. Zhu, X. He and J. Z. H. Zhang, Phys. Chem. Chem. Phys., 2012, 14, 7837-7845.

14 T. Zhu, J. Z. H. Zhang and Z. He, J. Chem. Theory Comput., 2013, 9, 2104-2114.

15 S. Tang and D. A. Case, J. Biomol. NMR, 2011, 51, 303-312.

16 A. Frank, I. Onila, H. M. Möller and T. E. Exner, Proteins: Struct., Funct., Bioinf., 2011, 79, 2189-2202.

17 A. Frank, H. M. Möller and T. E. Exner, J. Chem. Theory Comput., 2012, 8, 1480-1492.

18 T. E. Exner, A. Frank, I. Onila and H. M. Möller, J. Chem. Theory Comput., 2012, 8, 4818-4827.

19 Q. Gao, S. Yokojima, D. G. Fedorova, K. Kitaura, M. Sakurai and S. Nakamura, J. Chem. Theory Comput., 2010, 6, 1428-1444.

20 Q. Gao, S. Yokojima, D. G. Fedorov, K. Kitaura, M. Sakurai and S. Nakamura, Chem. Phys. Lett., 2014, 593, 165-173.

21 M. A. Collins, Phys. Chem. Chem. Phys., 2012, 14, 7744-7751.

22 M. A. Collins, M. W. Cvitkovic and R. P. A. Bettens, Acc. Chem. Res., 2014, 47, 2776-2785.

23 V. Deev and M. A. Collins, J. Chem. Phys., 2005, 122, 154102.

24 M. A. Addicoat and M. A. Collins, J. Chem. Phys., 2009, 131, 104103.
25 H. M. McConnell, J. Chem. Phys., 1957, 27, 226-230.

26 W. Li, S. Li and Y. Jiang, J. Phys. Chem. A, 2007, 111, 2193-2199.

27 X. Wang, J. Liu, J. Z. H. Zhang and X. He, J. Phys. Chem. A, 2013, 117, 7149-7161.

28 M. A. Collins, J. Chem. Phys., 2014, 141, 094108.

29 H.-A. Le, H.-J. Tan, J. F. Ouyang and R. P. A. Bettens, J. Chem. Theory Comput., 2012, 8, 469-478.

30 D. M. Reid and M. A. Collins, J. Chem. Phys., 2013, 139, 184117.

31 A. J. Stone, J. Chem. Theory Comput., 2005, 1, 1128-1132.

32 A. E. Reed and W. Frank, J. Chem. Phys., 1983, 4066-4073.

33 A. E. R. R. B. Weinstock and F. J. Weinhold, J. Chem. Phys., 1985, 83, 735-746.

34 F. H. Allen, Acta Crystallogr., Sect. B: Struct. Sci., 2002, 58, 380-388.

35 W. Hua, T. Fang, W. Li, J.-G. Yu and S. Li, J. Phys. Chem. A, 2008, 112, 10864-10872.

36 M. J. Frisch, G. W. Trucks, H. B. Schlegel, G. E. Scuseria, M. A. Robb, J. R. Cheeseman, G. Scalmani, V. Barone, B. Mennucci, G. A. Petersson, H. Nakatsuji, M. Caricato, X. Li, H. P. Hratchian, A. F. Izmaylov, J. Bloino, G. Zheng, J. L. Sonnenberg, M. Hada, M. Ehara, K. Toyota, R. Fukuda, J. Hasegawa, M. Ishida, T. Nakajima, Y. Honda, O. Kitao, H. Nakai, T. Vreven, J. A. Montgomery, Jr., J. E. Peralta, F. Ogliaro, M. Bearpark, J. J. Heyd, E. Brothers, K. N. Kudin, V. N. Staroverov, R. Kobayashi, J. Normand, K. Raghavachari, A. Rendell, J. C. Burant, S. S. Iyengar, J. Tomasi, M. Cossi, N. Rega, J. M. Millam, M. Klene, J. E. Knox, J. B. Cross, V. Bakken, C. Adamo, J. Jaramillo, R. Gomperts, R. E. Stratmann, O. Yazyev, A. J. Austin, R. Cammi, C. Pomelli, J. W. Ochterski, R. L. Martin, K. Morokuma, V. G. Zakrzewski, G. A. Voth, P. Salvador, J. J. Dannenberg, S. Dapprich, A. D. Daniels, O. Farkas, J. B. Foresman, J. V. Ortiz, J. Cioslowski and D. J. Fox, Gaussian 09 Revision C.1, Gaussian Inc., Wallingford CT, 2009.

37 F. Jensen, J. Chem. Theory Comput., 2008, 4, 719-727.

38 D. B. Chesnut and K. D. Moore, J. Comput. Chem., 1989, 10, 648-659.

39 D. B. Chesnut, B. E. Rusiloski, K. D. Moore and D. A. Egolf, J. Comput. Chem., 1993, 14, 1364-1375.

40 D. M. Reid, R. Kobayashi and M. A. Collins, J. Chem. Theory Comput., 2014, 10, 146-152. 